\title{
6D thick branes from interacting scalar fields
}

\author{
Vladimir Dzhunushaliev* \\ Department of Physics and Microel. Engineering, Kyrgyz-Russian Slavic University, \\ Bishkek, Kievskaya Str. 44, 720021, Kyrgyz Republic \\ Vladimir Folomeev ${ }^{\dagger}$ \\ Institute of Physics of NAS KR, 265 a, Chui str., Bishkek, 720071, Kyrgyz Republic \\ Douglas Singleton ${ }^{\ddagger}$ and Sergio Aguilar-Rudametkin ${ }^{\S}$ \\ Physics Department, CSU Fresno, Fresno, California 93740-8031, USA
}

(Received 1 May 2007; revised manuscript received 5 December 2007; published 4 February 2008)

\begin{abstract}
A thick brane in six dimensions is constructed using two scalar fields. The field equations for 6D gravity plus the scalar fields are solved numerically. This thick brane solution shares some features with previously studied analytic solutions, but has the advantage that the energy-momentum tensor which forms the thick brane comes from the scalar fields rather than being put in by hand. Additionally the scalar fields which form the brane also provide a universal, nongravitational trapping mechanism for test fields of various spins.
\end{abstract}

DOI: 10.1103/PhysRevD.77.044006

PACS numbers: 04.50.- $\mathrm{h}$

\section{INTRODUCTION}

The brane world scenario, where our four dimensional spacetime is seen as a lower dimensional membrane embedded in a higher dimensional spacetime was proposed in [1-3] (see [4] for earlier work) as a means of addressing the hierarchy problem. These brane models have also been used to investigate other open questions in particle physics and cosmology such as the fermion generation puzzle $[5,6]$ or the nature of dark energy [7] and dark matter [8].

In order to have an effective four dimensional spacetime in these brane world models one must have a method of confining or trapping particles/fields to a four-dimensional spacetime. The localization of fields of various spins has been investigated by various authors [9-12]. In particular Ref. [9] showed that it was not possible to trap spin 1 gauge bosons in the original 5D models of [2,3]. More specifically one could not trap spin 1 fields using gravity alone. One had to introduce some other interaction to trap spin 1 fields. Further in Ref. [10] it was found that if one arranged the parameters of the 5D brane models of [2,3] so that spin 0 and spin 2 fields were trapped then spin $\frac{1}{2}$ would be repelled from the brane, and conversely if one arranged the parameters so that $\operatorname{spin} \frac{1}{2}$ fields were trapped then the spin 0 and spin 2 field would be repelled.

In $[13,14]$ it was shown that by going from $5 \mathrm{D}$ to $6 \mathrm{D}$ it was possible to trap fields of all spins (i.e. spins $0, \frac{1}{2}, 1,2$ ) to a 4D spacetime using only gravity. The "trapping" provided by the $6 \mathrm{D}$ solution of [14] is automatic since, as we discuss shortly, the proper distance away from the brane

\footnotetext{
*dzhun@krsu.edu.kg

†vfolomeev@mail.ru

dougs@csufresno.edu

siarudametkin@hotmail.edu
}

at $r=0$ is finite so any field can only be some finite distance away from the brane. We briefly recall the features of this $6 \mathrm{D}$ brane solution that are relevant for the present paper.

First the $6 \mathrm{D}$ action was

$$
S=\int d^{6} x \sqrt{-g}\left[\frac{M^{4}}{2} R+\Lambda+L_{m}\right],
$$

where $M, R, \Lambda$, and $L_{m}$ are, respectively, the fundamental scale, the scalar curvature, the cosmological constant and the matter field Lagrangian. All of these physical quantities refer to six-dimensional spacetime with the signature $(+-$ $----)$.

Variation of the action (1) with respect to the sixdimensional metric tensor $g_{A B}$ led to Einstein's equations:

$$
R_{A B}-\frac{1}{2} g_{A B} R=\frac{1}{M^{4}}\left(g_{A B} \Lambda+T_{A B}\right),
$$

where $R_{A B}$ and $T_{A B}$ are the Ricci and the energymomentum tensors, respectively. Capital Latin indices run over $A, B, \ldots=0,1,2,3,5,6$.

The four-dimensional Einstein equations were taken as $R_{\mu \nu}^{(4)}-\frac{1}{2} \eta_{\mu \nu} R^{(4)}=0$ i.e. the ordinary vacuum equations without no cosmological constant. Greek indices $\alpha, \beta, \ldots=0,1,2,3$ refer to four dimensions. The source ansatz functions were

$$
T_{\mu \nu}=-g_{\mu \nu} F(r), \quad T_{i j}=-g_{i j} K(r), \quad T_{i \mu}=0 .
$$

Small Latin indices refer to the extra coordinates $i, j=5$, 6 . Finally, the metric was taken to have the form

$$
d s^{2}=\phi^{2}(r) \eta_{\mu \nu} d x^{\mu} d x^{\nu}-\lambda(r)\left(d r^{2}+r^{2} d \theta^{2}\right) .
$$

The metric of ordinary 4 -space, $\eta_{\alpha \beta}$, has the signature 
$(+,-,-,-)$. The 4D and 2D "warp" factor, ansatz functions $\phi(r)$ and $\lambda(r)$, depend only on the extra radial coordinate, $r$. The Einstein field Eqs. (2) in terms of the ansatz functions are [14]

$$
\begin{aligned}
3 \frac{\phi^{\prime \prime}}{\phi}+3 \frac{\phi^{\prime}}{r \phi}+3 \frac{\left(\phi^{\prime}\right)^{2}}{\phi^{2}}+\frac{1}{2} \frac{\lambda^{\prime \prime}}{\lambda}-\frac{1}{2} \frac{\left(\lambda^{\prime}\right)^{2}}{\lambda^{2}}+\frac{1}{2} \frac{\lambda^{\prime}}{r \lambda} \\
=\frac{\lambda}{M^{4}}[F(r)-\Lambda], \\
\frac{\phi^{\prime} \lambda^{\prime}}{\phi \lambda}+2 \frac{\phi^{\prime}}{r \phi}+3 \frac{\left(\phi^{\prime}\right)^{2}}{\phi^{2}}=\frac{\lambda}{2 M^{4}}[K(r)-\Lambda], \\
2 \frac{\phi^{\prime \prime}}{\phi}-\frac{\phi^{\prime} \lambda^{\prime}}{\phi \lambda}+3 \frac{\left(\phi^{\prime}\right)^{2}}{\phi^{2}}=\frac{\lambda}{2 M^{4}}[K(r)-\Lambda],
\end{aligned}
$$

where the prime $=\partial / \partial r$. These equations are for the $\alpha \alpha$, $r r$, and $\theta \theta$ components, respectively. The three Eqs. (5)(7) are not all independent and can be reduced to a set of two equations for $\phi(r), \lambda(r)$. We perform such a reduction in the following section. An analytic solution to these equations was found with the ansatz functions of the form

$$
\phi(r)=\frac{c^{2}+a r^{2}}{c^{2}+r^{2}} \stackrel{r \rightarrow \infty}{\rightarrow} a, \quad \lambda(r)=\frac{c^{4}}{\left(c^{2}+r^{2}\right)^{2}} \stackrel{r \rightarrow \infty}{\rightarrow} \frac{c^{4}}{r^{4}},
$$

where $c, a$ are constant. The source functions are

$$
\begin{aligned}
& F(r)=\frac{f_{1}}{2 \phi(r)^{2}}+\frac{3 f_{2}}{4 \phi(r)}, \stackrel{r \rightarrow \infty}{\rightarrow} \frac{f_{1}}{2 a^{2}}+\frac{3 f_{2}}{4 a}, \\
& K(r)=\frac{f_{1}}{\phi(r)^{2}}+\frac{f_{2}}{\phi(r)} \stackrel{r \rightarrow \infty}{\rightarrow} \frac{f_{1}}{a^{2}}+\frac{f_{2}}{a},
\end{aligned}
$$

where the constants $f_{1}=-\frac{3 \Lambda}{5} a$ and $f_{2}=\frac{4 \Lambda}{5}(a+1)$ are determined by the $6 \mathrm{D}$ cosmological constant, $\Lambda$, and the constant, $a$, from the 4D warp function $\phi(r)$.

A drawback of this solution is that the matter sources are put in by hand via the ansatz functions $F(r)$ and $K(r)$ rather than being given by some realistic field source. Also from $T_{\mu \nu}$ from (3) and $F(r)$ from (9) [as well as using the asymptotic values of $F(r)$ at $r=0$ and $r=\infty$ ] one sees that the energy density is negative on the brane and decreases to some negative, asymptotic, value at $r=\infty$. Other authors have used such "phantom" sources to construct brane world models [15]. In the present work we show that it is possible to replace the "by-hand" matter sources given by $F(r), K(r)$ by more realistic scalar field sources, and still obtain the 4D and 2D warp factors similar to those from [14]. We also find that the energy density coming from the scalar fields has better asymptotic behavior - the energy density, while still negative on the brane, approaches 0 as $r \rightarrow \infty$. Finally we give a new, simple, nongravitational trapping mechanism which works for test fields of any spin. This new trapping mechanism arises from one of the scalar fields which now replace the arbitrary source ansatz functions, $F(r), K(r)$.

\section{6D BRANE FROM SCALAR FIELDS}

We again start with 6D gravity and some matter source. The action is

$$
S=\int d^{6} x \sqrt{-g}\left[\frac{M^{4}}{2} R+L_{m}\right]
$$

where we have apparently dropped the 6D cosmological constant and the matter source is composed of two interacting scalar fields, $\chi(r)$ and $\varphi(r)$ with the Lagrangian

$$
L_{m}=\frac{1}{2} \partial_{A} \varphi \partial^{A} \varphi+\frac{1}{2} \partial_{A} \chi \partial^{A} \chi-V(\varphi, \chi),
$$

with the potential energy given by

$$
V(\varphi, \chi)=\frac{\Lambda_{1}}{4}\left(\varphi^{2}-m_{1}^{2}\right)^{2}+\frac{\Lambda_{2}}{4}\left(\chi^{2}-m_{2}^{2}\right)^{2}+\varphi^{2} \chi^{2}-V_{0} .
$$

Where the constant $V_{0}$ is set as $V_{0}=\left(\Lambda_{2} m_{2}^{4} / 4\right)$ and acts as a negative cosmological constant. In this paper the values of $\Lambda_{1}, \Lambda_{2}$ were taken as $\Lambda_{1}=0.1$ and $\Lambda_{2}=1.0$. For these values (and the associated $m_{1}, m_{2}$ which we find below) the potential (12) has two global minima at $\varphi=0, \chi= \pm m_{2}$ and two local minima at $\chi=0, \varphi= \pm m_{1}$. For all four of these points one finds $\partial_{\varphi} V(\varphi, \chi)=\partial_{\chi} V(\varphi, \chi)=0$. Also at these points $V\left( \pm m_{1}, 0\right)=0>V\left(0, \pm m_{2}\right)=\left(\Lambda_{1} m_{1}^{4} / 4\right)-$ $V_{0}$, which shows that the first two points are local minima. The fact that $0>V\left(0, \pm m_{2}\right)$ or $V_{0}>\Lambda_{1} m_{1}^{4} / 4$ depends on the, as yet undetermined, values of $m_{1}, m_{2}$. Below we find $m_{1}, m_{2}$ and they have values such that $V_{0}>\Lambda_{1} m_{1}^{4} / 4$ which makes the points $\varphi=0, \chi= \pm m_{2}$ global minima. Besides two local and two global minima, four unstable saddle points exist:

$$
\begin{aligned}
& \varphi= \pm \sqrt{\frac{\Lambda_{2}}{2}} \sqrt{m_{2}^{2}-\frac{\Lambda_{1} \Lambda_{2} m_{2}^{2}-2 \Lambda_{1} m_{1}^{2}}{\Lambda_{1} \Lambda_{2}-4}}, \\
& \chi= \pm \sqrt{\frac{\Lambda_{1} \Lambda_{2} m_{2}^{2}-2 \Lambda_{1} m_{1}^{2}}{\Lambda_{1} \Lambda_{2}-4}} .
\end{aligned}
$$

The solutions which we find in this paper go to the local minima, $\chi=0, \varphi= \pm m_{1}$, and are thus not absolutely stable. However, by adjusting parameters in the potential one can make the barrier between the local and global minima large enough so that tunneling between them is arbitrarily small, making the local minima metastable.

Other attempts to construct thick brane solutions from scalar fields can be found in [16-19]. In some sense, with (11) and (12) one is replacing the two ansatz function, $F(r), K(r)$ of the previous solution by two scalar fields. The two real scalar fields $\varphi, \chi$ depend only on the extra coordinate $r ; m_{1}, m_{2}$ are the masses of these fields and $\Lambda_{1}, \Lambda_{2}$ are the self-coupling constants. The effective, negative cosmological constant (i.e. the $V_{0}$ term in the 
potential) is the physical reason for the formation of the brane - the attraction of the ordinary matter is balanced by the repulsion coming from the negative cosmological constant. The potential in (12) was used in [20] as an approximate, effective description of a condensate of gauge field in SU(3) Yang-Mills theory i.e. the scalar fields were taken as effective fields describing condensates of Yang-Mills fields. In this view one can think of the brane as being formed from Yang-Mills fields whose condensates are effectively represented by the scalar fields. The general field equations for the scalar fields are given by

$$
\frac{1}{\sqrt{-{ }^{6} g}} \frac{\partial}{\partial x^{A}}\left[\sqrt{-{ }^{6} g} g^{A B} \frac{\partial(\varphi, \chi)}{\partial x^{B}}\right]=-\frac{\partial V}{\partial(\varphi, \chi)} .
$$

For the 6D metric given by (4) the $\varphi, \chi$ scalar field equations become

$$
\begin{aligned}
& \varphi^{\prime \prime}+\left(\frac{1}{r}+4 \frac{\phi^{\prime}}{\phi}\right) \varphi^{\prime}=\lambda \varphi\left[2 \chi^{2}+\Lambda_{1}\left(\varphi^{2}-m_{1}^{2}\right)\right], \\
& \chi^{\prime \prime}+\left(\frac{1}{r}+4 \frac{\phi^{\prime}}{\phi}\right) \chi^{\prime}=\lambda \chi\left[2 \varphi^{2}+\Lambda_{2}\left(\chi^{2}-m_{2}^{2}\right)\right] .
\end{aligned}
$$

The Einstein field equations for the metric ansatz functions have the same left-hand side as in (5), but now the energymomentum tensor on the right-hand side comes from the two scalar fields. The general form for the scalar field energy-momentum tensor is

$$
T_{A B}=\frac{\partial L_{m}}{\partial \varphi^{, A}} \varphi_{, B}+\frac{\partial L_{m}}{\partial \chi^{\prime A}} \chi_{, B}-g_{A B} L_{m} .
$$

The specific components of the energy-momentum tensor are

$$
\begin{aligned}
T_{\mu \nu} & =g_{\mu \nu}\left[\frac{1}{2 \lambda}\left(\varphi^{\prime 2}+\chi^{\prime 2}\right)+V(\varphi, \chi)\right] \\
T_{r r} & =-\lambda\left[-\frac{1}{2 \lambda}\left(\varphi^{\prime 2}+\chi^{\prime 2}\right)+V(\varphi, \chi)\right], \\
T_{\theta \theta} & =-r^{2} \lambda\left[\frac{1}{2 \lambda}\left(\varphi^{\prime 2}+\chi^{\prime 2}\right)+V(\varphi, \chi)\right] .
\end{aligned}
$$

The Einstein field equations now become

$$
\begin{gathered}
3 \frac{\phi^{\prime \prime}}{\phi}+3 \frac{\phi^{\prime}}{r \phi}+3 \frac{\left(\phi^{\prime}\right)^{2}}{\phi^{2}}+\frac{1}{2} \frac{\lambda^{\prime \prime}}{\lambda}-\frac{1}{2} \frac{\left(\lambda^{\prime}\right)^{2}}{\lambda^{2}}+\frac{1}{2} \frac{\lambda^{\prime}}{r \lambda} \\
=-\frac{\lambda}{M^{4}}\left[\frac{1}{2 \lambda}\left(\varphi^{\prime 2}+\chi^{\prime 2}\right)+V(\varphi, \chi)\right], \\
\frac{\phi^{\prime} \lambda^{\prime}}{\phi \lambda}+2 \frac{\phi^{\prime}}{r \phi}+3 \frac{\left(\phi^{\prime}\right)^{2}}{\phi^{2}}=-\frac{\lambda}{2 M^{4}}\left[-\frac{1}{2 \lambda}\left(\varphi^{\prime 2}+\chi^{\prime 2}\right)\right. \\
+V(\varphi, \chi)],
\end{gathered}
$$

$$
\begin{aligned}
2 \frac{\phi^{\prime \prime}}{\phi}-\frac{\phi^{\prime} \lambda^{\prime}}{\phi \lambda}+3 \frac{\left(\phi^{\prime}\right)^{2}}{\phi^{2}}= & -\frac{\lambda}{2 M^{4}}\left[\frac{1}{2 \lambda}\left(\varphi^{\prime 2}+\chi^{\prime 2}\right)\right. \\
& +V(\varphi, \chi)] .
\end{aligned}
$$

We can reduce these three equations to two by multiplying (20) by $\frac{3}{2}$ and subtracting from (18) to get a second order differential equation for $\lambda(r)$; by subtracting (19) from (20) we get a second order differential equation for $\phi(r)$ :

$$
\begin{gathered}
\frac{\lambda^{\prime \prime}}{\lambda}-\left(\frac{\lambda^{\prime}}{\lambda}\right)^{2}-3\left(\frac{\phi^{\prime}}{\phi}\right)^{2}+3 \frac{\phi^{\prime}}{\phi} \frac{\lambda^{\prime}}{\lambda}+\frac{1}{r}\left(6 \frac{\phi^{\prime}}{\phi}+\frac{\lambda^{\prime}}{\lambda}\right) \\
=-\frac{1}{2} \lambda\left[\frac{1}{2 \lambda}\left(\varphi^{\prime 2}+\chi^{\prime 2}\right)+V(\varphi, \chi)\right], \\
\frac{\phi^{\prime \prime}}{\phi}-\frac{\phi^{\prime}}{\phi} \frac{\lambda^{\prime}}{\lambda}-\frac{\phi^{\prime}}{r \phi}=-\frac{1}{4}\left(\varphi^{\prime 2}+\chi^{\prime 2}\right) .
\end{gathered}
$$

The fundamental 6D gravity scale, $M$, has been adsorbed via the rescaling: $r \rightarrow r / M^{2}, \quad \varphi \rightarrow M^{2} \varphi, \quad \chi \rightarrow M^{2} \chi$, $m_{1,2} \rightarrow M^{2} m_{1,2}$.

We now show that the system of coupled, nonlinear differential Eqs. (14), (15), (21), and (22) have solutions which roughly share common features with the analytic brane solution given by (8) and (9). Unlike the system of Eqs. (5)-(7) we were not able find an analytical solution, but rather we solved the system numerically using the NDSolve routine from Mathematica. As with the solutions in (8) and (9) we require that the solution be 6D Minkowski on the brane $(r=0)$ so that $\phi(0)=1$ and $\lambda(0)=1$. For initial condition we chose the ansatz functions at $r=0$ as

$$
\begin{aligned}
& \varphi(0)=\sqrt{3}, \quad \varphi^{\prime}(0)=0, \quad \chi(0)=\sqrt{0.6}, \quad \chi^{\prime}(0)=0, \\
& \phi(0)=1.0, \quad \phi^{\prime}(0)=0, \quad \lambda(0)=1.0, \quad \lambda^{\prime}(0)=0 .
\end{aligned}
$$

Because of terms like $1 / r$ we started the NDSolve routine from $r=0.001$. Taking into account the vanishing of the first derivatives of all the ansatz functions from (23), all the ansatz functions had an expansion of the form $f(r)=$ $f(0)+f^{\prime \prime}(0) r^{2} / 2$. The terms $f^{\prime \prime}(0)$ were determined from one of the Eqs. (14), (15), and (18) or (20). For example $\phi(0.001)=\phi(0)+\frac{1}{2}(0.001)^{2} \phi^{\prime \prime}(0) \quad$ and $\quad \phi^{\prime}(0.001)=$ $(0.001) \phi^{\prime \prime}(0)$ where $\phi^{\prime \prime}(0)=-\frac{1}{4} \phi(0) \lambda(0) V(\varphi(0), \chi(0))$ was obtained from (12), (20), and (23). The scalar field self couplings were taken as $\Lambda_{1}=0.1$ and $\Lambda_{2}=1.0$. Once these initial conditions and scalar field self couplings were set we searched for solutions which had good asymptotic behavior i.e. we wanted the fields and metric warp factor functions to approach some constant, finite value as $r \rightarrow$ $\infty$. Such asymptotic conditions were only fulfilled for specific values of $m_{1}, m_{2}$. For the initial conditions given in (23) and for the chosen $\Lambda_{1}, \Lambda_{2}$ we found that $m_{1} \approx$ 2.462065 and $m_{2} \approx 3.0168291$ gave the desired asymptotic behavior. These values of the masses, which gave the 
asymptotically well-behaved ansatz functions, where found using the procedure outlined in [21]. The numerical solutions for the scalar fields and the metric warp factor functions using the initial conditions in (23) are shown in Figs. 1 and 2, respectively. Note that since the warp factors start as $\phi(0)=\lambda(0)=1$ one has 6D Minkowski spacetime on the brane, $r=0$. As $r \rightarrow \infty \lambda(r) \rightarrow 0$ as $1 / r^{\alpha}$ while $\phi(0)$ approaches some constant asymptotic value $>1$. These features are generally similar to the warp factors of the analytic solution given in (8). However, from Fig. 2 one sees that for the present case $\lambda(r)$ goes to zero as $r^{-0.45}$ which is a much slower falloff compared to the $\lambda(r)$ given in (8). The scalar fields approach the asymptotic values $\varphi(\infty)=m_{1}$ and $\chi(\infty)=0$. Looking at the potential, $V(\varphi, \chi)$, from (12) one sees that $V\left(\varphi(\infty)=m_{1}, \chi(\infty)=\right.$ $0)=0>V\left(\varphi(\infty)=0, \chi(\infty)=m_{2}\right)=-V_{0}$. Thus the solution is not absolutely stable since it sits at a local rather than global minimum. However, by adjusting parameters in the potential $V(\varphi, \chi)$ one can make the barrier between the local and global minimum large. Thus tunneling between the local and global minimum will be unlikely making this state with $\varphi(\infty)=m_{1}$ and $\chi(\infty)=0$ effectively stable over a long time scale e.g. long compared to the age of the Universe.

Once the scalar fields, $\varphi(r), \chi(r)$, are known, it is possible to calculate their energy density. Plugging the numerical solutions for the scalar fields into (17) the energy density is given by Fig. 3. Both the energy density of the scalar fields system, given in Fig. 3, and the energy density of the analytical solution, given by Eqs. (3) and (9), approached a constant value as $r \rightarrow \infty$. However, for the scalar fields case the asymptotic value was zero, while for the analytic solution it was a negative constant. Both solutions have some range of $r$, for which $T_{00}<0$ - for the scalar fields this region is only near the brane (see

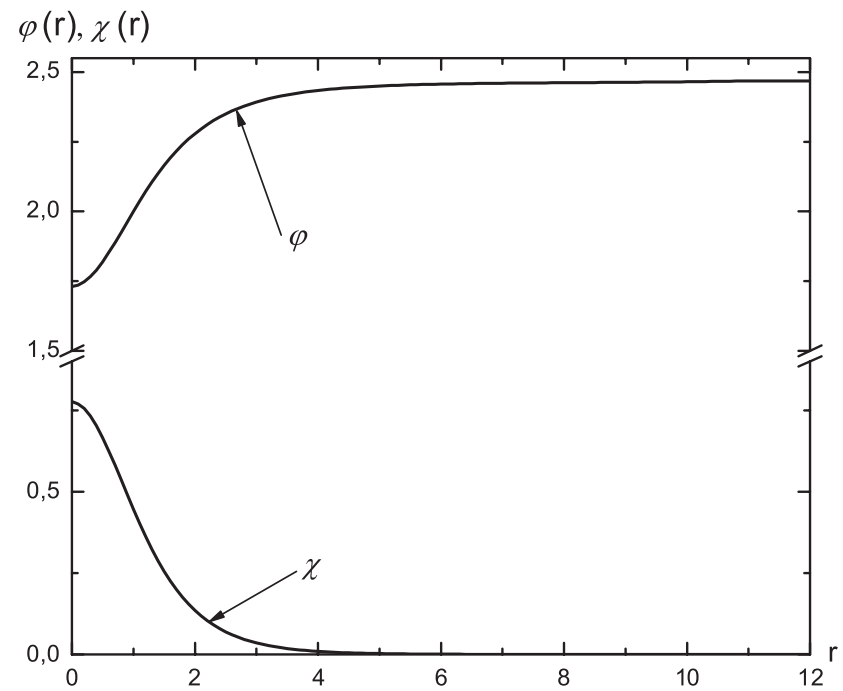

FIG. 1. The scalar fields $\varphi, \chi$ for the initial conditions given in (23).

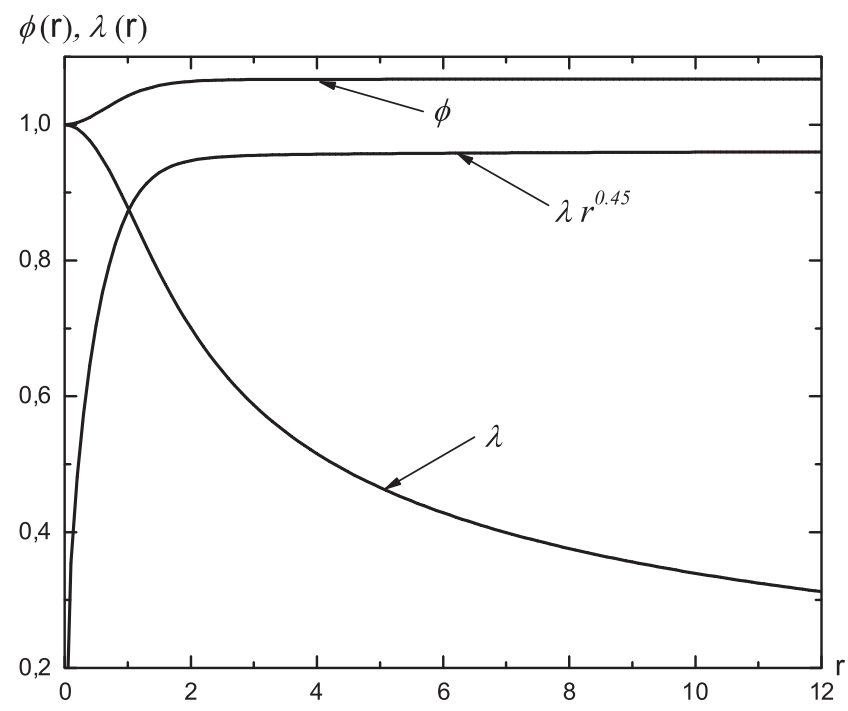

FIG. 2. The metric functions $\phi, \lambda$ for the initial conditions given in (23). The middle curve indicates the power law falloff for $\lambda \approx r^{-0.45}$.

Fig. 3) while for the analytic solution $T_{00}<0$ for all $r$. In the scalar fields case this negative energy density may provide a physical explanation for the formation of the brane: the negative energy density provides a repulsive force which can balance the usual attraction due to gravity.

We now analyze the asymptotic forms for the solutions to the scalar field equations, (14) and (15), and the Einstein field equations, (18)-(20). The asymptotic forms of the ansatz functions can be written as

$$
\begin{aligned}
& \varphi \approx m_{1}-\delta \varphi(r), \quad \chi \approx \delta \chi(r), \\
& \phi \approx \phi_{\infty}, \quad \lambda \approx \frac{\gamma}{r^{\alpha}} .
\end{aligned}
$$

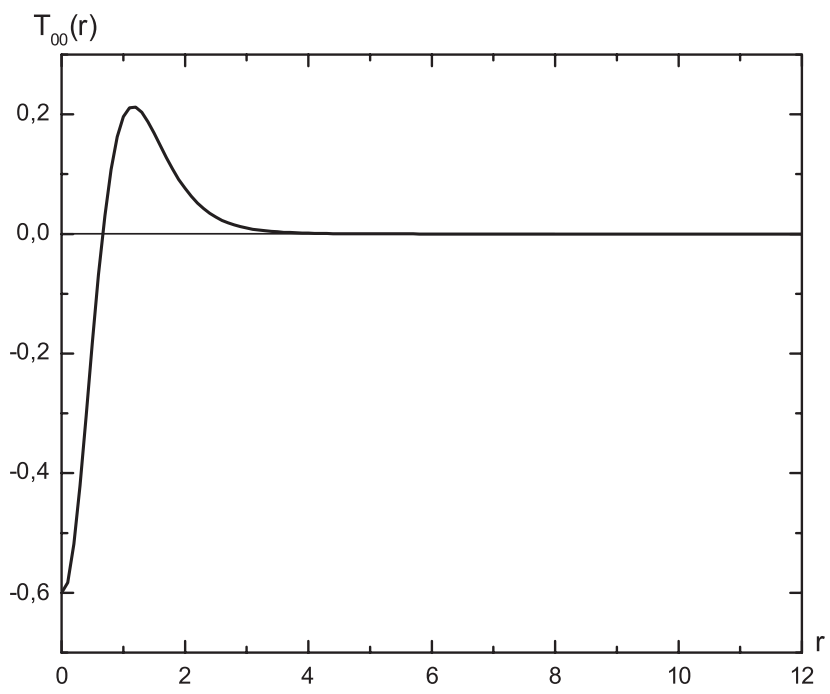

FIG. 3. The energy density $T_{00}(r)$ from (17). $T_{00}(r)<0$ for $r<1$. 
Where $\delta \varphi(r), \delta \chi(r) \ll 1$ as $r \rightarrow \infty$, and $\gamma$ is some dimensionful constant. The subscript " $\infty$ " indicates the asymptotic value of $\phi(r)$. Note, in particular, that the 2D metric ansatz function, $\lambda(r)$ has a nontrivial behavior as $r^{-\alpha}$. This comes about since this form gives zero when used in the three terms involving $\lambda$ and its derivatives on the left-hand side of (18). The three terms involving $\phi$ and its derivatives on the left-hand side of (18) yields to a similar analysis, but the minus sign difference in this case results in the exponent being zero so that $\phi \approx r^{0}=$ constant. From the numerical analysis - see Fig. 2-the exponent for the 2D metric ansatz function is $\alpha=0.45$ so that $\lambda \approx \gamma / r^{-0.45}$. One can see-ignoring terms that are second order in small quantities like $\varphi^{\prime}(r), \chi, \chi^{\prime}(r)$ - that the Einstein field equations given by (18)-(20) are satisfied in the asymptotic forms in (24). The right-hand sides of (18)-(20) go to zero since both $\varphi^{\prime 2}, \chi^{\prime 2} \rightarrow 0$ and $V(\varphi, \chi) \rightarrow 0$ as $r \rightarrow \infty$ for the asymptotic forms given in (24). Furthermore, one can see the left-hand sides also go to zero for the asymptotic forms for $\phi(r), \lambda(r)$ given in (24).

Turning to the asymptotic form for the scalar field equations - (14) and (15) — we find that for the asymptotic forms from (24) these equations become

$$
\begin{aligned}
& \delta \varphi^{\prime \prime}+\frac{1}{r} \delta \varphi^{\prime}=\frac{2 \gamma \Lambda_{1} m_{1}^{2}}{r^{\alpha}} \delta \varphi, \\
& \delta \chi^{\prime \prime}+\frac{1}{r} \delta \chi^{\prime}=\frac{\gamma\left(2 m_{1}^{2}-\Lambda_{2} m_{2}^{2}\right)}{r^{\alpha}} \delta \chi,
\end{aligned}
$$

These equations have the solutions

$$
\begin{gathered}
\delta \varphi=C_{\varphi} K_{0}\left(\frac{2 \sqrt{2 \gamma \Lambda_{1} m_{1}^{2}}}{2-\alpha} r^{1-\alpha / 2}\right) \\
\approx C_{\varphi} \frac{\exp \left(-\frac{2 \sqrt{2 \gamma \Lambda_{1} m_{1}^{2}}}{2-\alpha} r^{1-\alpha / 2}\right)}{\sqrt{r^{1-\alpha / 2}}}, \\
\delta \chi=C_{\chi} K_{0}\left(\frac{2 \sqrt{\gamma\left(2 m_{1}^{2}-\Lambda_{2} m_{2}^{2}\right)}}{2-\alpha} r^{1-\alpha / 2}\right) \\
\approx C_{\chi} \frac{\exp \left(-\frac{2 \sqrt{2 \gamma\left(2 m_{1}^{2}-\Lambda_{2} m_{2}^{2}\right)}}{2-\alpha} r^{1-\alpha / 2}\right)}{\sqrt{r^{1-\alpha / 2}}},
\end{gathered}
$$

where $\mathrm{K}_{0}(\mathrm{Cr})$ are the zeroth order modified Bessel function of second kind, which asymptotically decay as $e^{-C r} / \sqrt{r}$. This analysis indicates that asymptotically the ansatz functions are well behaved, and this analytic, asymptotic analysis matches the numerical solutions shown in Figs. 1 and 2.

From (26) and (27), one can see that it is necessary to take $\alpha<2$ in order that the scalar fields decay as $r \rightarrow \infty$. The numerical analysis in our case gives $\alpha \approx 0.45$. Thus as indicated in Fig. 1 and confirmed by the asymptotic forms with $\alpha \approx 0.45$ we do have decaying solutions for the scalar fields (in the case of the $\varphi$ field it is "decaying" to a nonzero constant).

One final, and important, difference between the thick brane solution of the present section and the analytic solution of the previous section is that the proper distance for the present solution is infinite. This can be seen directly by calculating the proper length

$$
l=\int_{0}^{r_{0}} \sqrt{\lambda(r)} d r \approx \int_{0}^{r_{0}} \sqrt{\frac{\gamma}{r^{0.45}}} d r \stackrel{r_{0} \rightarrow \infty}{\rightarrow} \infty
$$

which is infinite. For the analytic solution of the previous section $\lambda(r) \rightarrow c^{4} / r^{4}$ from (8). Using this asymptotic form in (28) gives a finite proper length.

Because the proper length away from the brane is infinite rather than finite-as was the case for the analytic solution of (8) - we need to revisit the question of whether fields of various spins are trapped or not. For the analytic solution of (8) this question was investigated in detail in $[13,14]$ and not unsurprisingly all the various spin fields were "trapped" exactly because the proper length was finite. The criteria for trapping taken in $[13,14]$ was that the integration of the action for some test field of spin $0, \frac{1}{2}$, 1, 2 over the extra dimensions should be finite. This is the criteria for trapping we take in the present work. One can also take as the criteria for trapping that total field energy per unit 3-volume of the brane (i.e. the integral of the energy density, $T_{0}{ }^{0}$, over the extra spatial dimensions) be finite [16]. In the present work these two criteria give the same result in regard to whether or not the field is trapped for all cases we consider.

We now investigate in detail the trapping of a spin 0 field. The spin 1 and spin 2 cases are identical to the spin 0 case. The spin $\frac{1}{2}$ case can be worked out along the same lines as the other fields using the setup given in [13]. The details are somewhat different than for the integer spin fields but the final conclusion - that the spin $\frac{1}{2}$ and all other spin fields are not trapped-is the same. We begin by considering a real scalar field $S\left(x^{A}\right)$ with an action given by

$$
S_{0}=-\frac{1}{2} \int d^{6} x g^{M N} \partial_{M} S \partial_{N} S
$$

The field equations for $S\left(x^{A}\right)$ resulting from (29) are similar to those for $\varphi, \chi(13)$ but without a potential term

$$
\frac{1}{\sqrt{-{ }^{6} g}} \partial_{A}\left[\sqrt{-{ }^{6} g} g^{M N} \partial_{N} S\left(x^{A}\right)\right]=0
$$

Making a separation of variables as $S\left(x^{A}\right)=\Sigma\left(x^{\mu}\right) s(r)$ one finds [22] that (30) is solved by $s(r)=C_{0}=$ const and $\eta^{\mu \nu} \partial_{\mu} \partial_{\nu} \Sigma=0$ i.e. the 4D scalar wave function is massless. Inserting this solution into the action (29) one obtains 


$$
\begin{aligned}
S_{0}= & -\pi \int_{0}^{\infty} d r\left[r \phi^{2}(r) \lambda(r)\right] \\
& \times \int d^{4} x \sqrt{-\eta}\left[\eta^{\mu \nu} \partial_{\mu} S \partial_{\nu} S\right]^{r \rightarrow \infty} \rightarrow \\
& -\pi C_{0}^{2} \phi_{\infty}^{2} \gamma \int_{0}^{\infty} r^{0.55} d r \int d^{4} x \sqrt{-\eta}\left[\eta^{\mu \nu} \partial_{\mu} \Sigma \partial_{\nu} \Sigma\right]
\end{aligned}
$$

where we have used the asymptotic forms $\phi(\infty)=\phi_{\infty}$ and $\lambda(\infty)=\gamma / r^{0.45}$. The integral over $r$ in the last line of (31) is divergent at large $r$. Thus the test scalar field is not trapped by the metric given by $\phi(r)$ and $\lambda(r)$. The cases of spin 1 and spin 2 fields also yield integrals which diverge as $\int_{0}^{\infty} r^{0.55} d r$. The spin $1 / 2$ case is different in detail but also yields a divergent integral which goes as $\int_{0}^{\infty} r^{-0.33} d r$. Thus none of the fields are trapped by the metric alone. In the next section we show that the exponential form for the scalar field $\chi(r)$ can give a universal trapping mechanism.

Before moving on to the trapping mechanism we make a few comments about the geometric character of the metric given by Fig. 2 and by the asymptotic expressions (24). The metric solution is a co-dimension 2 object which is asymptotically flat as $r \rightarrow \infty$ and has a deficit angle of $2 \pi\left(1-\sqrt{1-\frac{\alpha}{2}}\right)$. To see this we change the asymptotic form of the metric [given by inserting (24) in (4)] via the transformation $R=r^{1-(\alpha / 2)}$. With this the 2D part of the metric becomes

$$
d s_{2 D}^{2} \rightarrow \frac{\gamma}{1-\frac{\alpha}{2}}\left(d R^{2}+\left(1-\frac{\alpha}{2}\right) R^{2} d \theta^{2}\right)
$$

For this 2D metric one defines a new angle $\bar{\theta}=\theta \sqrt{1-\frac{\alpha}{2}}$ so that $0 \leq \bar{\theta} \leq 2 \pi \sqrt{1-\frac{\alpha}{2}}$ which gives a deficit angle of $2 \pi\left(1-\sqrt{1-\frac{\alpha}{2}}\right)$. Since $\alpha=0.45$ this is a deficit angle rather than a proficit angle. Recently there has been interest in such brane world models with deficit [23] and proficit [24] angles to investigate open questions such as the cosmological constant and the fermion generation puzzle.

\section{TRAPPING MECHANISM}

All the test fields studied in the previous subsections have divergent actions coming from the integration over $r$. Thus none of these fields are trapped to the brane at $r=0$ and as it stands the model is not viable. However, the form of the scalar field, $\chi(r)$, provides a mechanism for trapping all the fields studied in the previous subsections. Note that all the spin fields have $r$ integrals that diverge as some power $\left(r^{1.55}\right.$ for spin 0 , spin 1 , and spin $2 ; r^{0.67}$ for $\left.\operatorname{spin} \frac{1}{2}\right)$. On the other hand, from (27) one sees that $\chi(r)$ decreases exponentially. This exponential decrease depends on several things: (i) $\gamma>0$, (ii) $m_{1}^{2}>\frac{\Lambda_{2} m_{2}^{2}}{2}$, (iii) $\alpha<2$. If (i) or (ii) is not satisfied then $\chi(r)$ is oscillatory; if (ii) is not satisfied $\chi(r)$ grows exponentially. For our solution all three conditions are met so $\chi(r)$ decreases exponentially.
Since the decrease of $\chi(r)$ is exponential while the divergence of the $r$ integration for each of the fields is some power law $r^{b}$ with $b<1$ one can get the $r$ integration to converge by multiplying the Lagrangian density of each spin field by some positive power of $\chi(r)$ as

$$
S_{s}=\int d^{6} x \chi^{n}(r) L_{s}
$$

where $n>0$ and $s=0, \frac{1}{2}, 1,2$. Redoing the analysis of the previous subsections it is easy to see that this procedure will make each of the $r$ integrations converge thus giving a trapping of the test field.

An even more stringent and less ad hoc trapping mechanism can be obtained by introducing a dilatonlike exponential coupling as suggested in the seminal paper $[25,26]$. As in these works we can introduce an exponential, dilatonlike coupling between $\chi(r)$ and the various spin fields in the following way:

$$
S_{s}=\int d^{6} x\left(1-e^{-2 \chi(r)}\right) L_{s}
$$

Since $\chi(r)$ exponentially goes to zero the factor $1-e^{-2 \chi(r)}$ will make the $r$ integration for each of the various spin test fields strongly convergent. In each case (spin $0, \frac{1}{2}, 1,2$ ) we find the fields are exponentially trapped by the behavior of $\chi(r)$ to some small region near the brane at $r=0$.

The nongravitational, trapping mechanism suggested by (32) or (33) is simple and universal. It is made possible by the asymptotic behavior of the scalar field $\chi(r)$ which plays a dual role of forming the brane and trapping test fields of all spin to the brane. This mechanism may be compared in some respects to confinement in quantum chromodynamics or to the confinement of magnetic charges inside a superconductor. Particularly in the magnetic charges inside a superconductor example, the scalar field condensate of Cooper pairs plays a crucial role.

\section{CONCLUSIONS}

We have constructed a thick brane solution in 6D spacetime using two self-interacting and mutually interacting scalar fields. This thick brane solution had the same general characteristics as the analytic solution given (8) and (9): the warp factors, $\phi(r), \lambda(r)$ were equal to 1 at $r=0$ so that on the brane one had a 6D Minkowski spacetime. As $r \rightarrow \infty$ the warp factor functions approached constant asymptotic values $\phi(\infty)>1$ and $\lambda(\infty)=0$. The energy densities were also similar-both were negative on the brane and approached some different, asymptotic value as $r \rightarrow \infty$. Two important advantages of the present solution is that the asymptotic value of the energy density in the bulk was zero rather than negative. In both cases the negative energy density may provide a physical explanation for the formation of the brane - the repulsion from the negative energy density can balance the attraction due to gravity. Another advantage of the present solution is that 
the energy-momentum tensor for the previous analytic solution was "fixed by hand" in order to give the warp factors in (8) which provided the universal gravitational trapping of particles/fields of all spins. In the present case the energy-momentum tensor comes from a more realistic source i.e. two scalar fields. In essence the two ansatz functions $F(r), K(r)$ from (9) have been replaced by the two scalar fields $\varphi(r), \chi(r)$.

Because the 2D warp factor, $\lambda(r)$, goes to zero as $r \rightarrow \infty$ according to a power law (24) with $\alpha<2$, the proper distance away from the brane is infinite, rather than finite as in the case of the analytic solution in [14]. Thus we were forced to introduce a new, simple, nongravitational trapping mechanism for test fields of various spins moving in the background (4) with $\phi(r), \lambda(r)$ given by Fig. 2 . The mechanism involves multiplying the test field Lagrange density for a test field of spin $s$ by some positive power of the scalar field $\chi(r)$ as in (32) and (33). Thus $\chi(r)$ not only forms the brane near $r=0$ with an energy density peaked near $r=0$ (see Fig. 3) and going to zero at $r=\infty$, but also is responsible for the trapping of test fields of various spins to the brane.
The brane solution found here is not absolutely stable since it settles into one of the local minima at $\varphi(\infty)=m_{1}$ and $\chi(\infty)=0$ rather than one of the global minima at $\varphi(\infty)=0$ and $\chi(\infty)= \pm m_{2}$. However, the barrier between the local and global minima can be made large by adjusting the parameters of the scalar potential (12). For example, one could chose different values of $\Lambda_{1}, \Lambda_{2}$. In this way the decay probability of the present solution from the local minimum to the global minimum via tunneling could be made small. This would make the brane solution presented here effectively stable over long time scales. One important, related question not studied in the present paper is the stability of this solution in the Lyapunov sense (see for example [27]). We leave this question for future study.

\section{ACKNOWLEDGMENTS}

V.D. acknowledges D. Singleton for the invitation to do research at Fresno State University and the support of a CSU Fresno Provost grant. D. S. acknowledges the support of a 2007 Summer Professional Development Grant from the CSM of CSU Fresno.
[1] N. Arkani-Hamed, S. Dimopoulos, and G. Dvali, Phys. Lett. B 429, 263 (1998); I. Antoniadis, N. Arkani-Hamed, S. Dimopoulos, and G. Dvali, Phys. Lett. B 436, 257 (1998).

[2] M. Gogberashvili, Int. J. Mod. Phys. D 11, 1635 (2002); 11, 1639 (2002); Europhys. Lett. 49, 396 (2000); Mod. Phys. Lett. A 14, 2025 (1999).

[3] L. Randall and R. Sundrum, Phys. Rev. Lett. 83, 3370 (1999); 83, 4690 (1999).

[4] K. Akama, in Gauge Theory and Gravitation, edited by K. Kikkawa, N. Nakanishi, and H. Nariai, Lecture Notes in Physics Vol. 176 (Springer-Verlag, Nara, Japan, 1982); V. A. Rubakov and M. E. Shaposhnikov, Phys. Lett. 125B, 136 (1983); 125B, 139 (1983); M. Visser, Phys. Lett. 159B, 22 (1985); G. W. Gibbons and D.L. Wiltshire, Nucl. Phys. B287, 717 (1987); I. Antoniadis, Phys. Lett. B 246, 377 (1990).

[5] N. Arkani-Hamed and M. Schmaltz, Phys. Rev. D 61, 033005 (2000); A. Mirabelli and M. Schmaltz, Phys. Rev. D 61, 113011 (2000).

[6] S. Aguilar and D. Singleton, Phys. Rev. D 73, 085007 (2006).

[7] C. Deffayet, G. R. Dvali, and G. Gabadadze, Phys. Rev. D 65, 044023 (2002); M. Gogberashvili, Phys. Lett. B 636, 147 (2006).

[8] M. Gogberashvili and M. Maziashvili, Gen. Relativ. Gravit. 37, 1129 (2005).

[9] A. Pomarol, Phys. Lett. B 486, 153 (2000).

[10] B. Bajc and G. Gabadadze, Phys. Lett. B 474, 282 (2000).

[11] A. Chodos and E. Poppitz, Phys. Lett. B 471, 119 (1999); A. G. Cohen and D. B. Kaplan, Phys. Lett. B 470, 52
(1999); R. Gregory, Phys. Rev. Lett. 84, 2564 (2000); Z. Chacko and A.E. Nelson, Phys. Rev. D 62, 085006 (2000); I. Oda, Phys. Rev. D 62, 126009 (2000); P. Kanti, R. Madden, and K. A. Olive, Phys. Rev. D 64, 044021 (2001); S. Randjbar-Daemi and $M$. Shaposhnikov, Nucl. Phys. B645, 188 (2002); M. Gogberashvili, Phys. Lett. B 553, 284 (2003); J. Math. Phys. (N.Y.) 43, 4886 (2002).

[12] T. Gherghetta and M. Shaposhnikov, Phys. Rev. Lett. 85, 240 (2000); T. Gherghetta, E. Roessl, and M. Shaposhnikov, Phys. Lett. B 491, 353 (2000).

[13] M. Gogberashvili and D. Singleton, Phys. Rev. D 69, 026004 (2004).

[14] M. Gogberashvili and D. Singleton, Phys. Lett. B 582, 95 (2004).

[15] R. Koley and S. Kar, Mod. Phys. Lett. A 20, 363 (2005); Classical Quantum Gravity 24, 79 (2007); arXiv:hep-th/ 0701266.

[16] K. Bronnikov and B. E. Meierovich, Gravitation Cosmol. 9, 313 (2003); S. Abdyrakhmanov, K. Bronnikov, and B. E. Meierovich, Gravitation Cosmol. 11, 82 (2005).

[17] N. Barbosa-Cendejas and A. Herrera-Aguilar, J. High Energy Phys. 10 (2005) 101; O. DeWolfe, D.Z. Freedman, S. S. Gubser, and A. Karch, Phys. Rev. D 62, 046008 (2000).

[18] R. Guerrero, R. Rodriguez, R. Ortiz, and R. Torrealba, Gen. Relativ. Gravit. 38, 845 (2006); R. Guerrero, R. Rodriguez, and R. Torrealba, Phys. Rev. D 72, 124012 (2005); R. Guerrero, A. Melfo, N. Pantoja, and R. Rodriguez, Phys. Rev. D 74, 084025 (2006).

[19] D. Bazeia and A. R. Gomes, J. High Energy Phys. 05 
(2004) 012; D. Bazeia, J. Menezes, and R. Menezes, Phys. Rev. Lett. 91, 241601 (2003).

[20] V. Dzhunushaliev, arXiv:hep-ph/0605070.

[21] V. Dzhunushaliev, Hadronic J. Suppl. 19, 185 (2004).

[22] I. Oda, Phys. Lett. B 571, 235 (2003).

[23] I. Navarro, Classical Quantum Gravity 20, 3603 (2003); S. M. Carroll and M. M. Guica, arXiv:hep-th/0302067; J. Vinet and J. M. Cline, Phys. Rev. D 70, 083514 (2004).
[24] M. Gogberashvili, P. Midodashvili, and D. Singleton, J. High Energy Phys. 08 (2007) 033.

[25] C. G. Callan, S.B. Giddings, J.A. Harvey, and A. Strominger, Phys. Rev. D 45, R1005 (1992).

[26] J. G. Russo, L. Susskind, and L. Thorlacius, Phys. Lett. B 292, 13 (1992).

[27] V. Rubakov, Classical Theory of Gauge Fields (Princeton University Press, Princeton, NJ, 2002), p. 143. 